Itinéraires Itinéraires

Littérature, textes, cultures

2020-3 | 2021

Le rap, une poésie de performances

\title{
Esthétique et performativité de la race dans "Créature ratée » de Casey
}

Aesthetics and the Performativity of Race in "Créature ratée" by Casey

\section{Emily Q. Shuman}

\section{(2) OpenEdition}

1 Journals

Édition électronique

URL : https://journals.openedition.org/itineraires/8805

DOI : $10.4000 /$ itineraires. 8805

ISSN : 2427-920X

Éditeur

Pléiade

\section{Référence électronique}

Emily Q. Shuman, «Esthétique et performativité de la race dans « Créature ratée » de Casey »,

Itinéraires [En ligne], 2020-3 | 2021, mis en ligne le 10 décembre 2021, consulté le 12 décembre 2021.

URL : http://journals.openedition.org/itineraires/8805; DOI : https://doi.org/10.4000/itineraires.8805

Ce document a été généré automatiquement le 12 décembre 2021.

\section{(c) (i) (9)}

Itinéraires est mis à disposition selon les termes de la licence Creative Commons Attribution - Pas d'Utilisation Commerciale - Pas de Modification 4.0 International. 


\title{
Esthétique et performativité de la race dans «Créature ratée » de Casey
}

Aesthetics and the Performativity of Race in "Créature ratée" by Casey

\author{
Emily Q. Shuman
}

1 Le rap trouve sa puissance esthétique dans plusieurs dimensions : les paroles, l'oralité de l'interprétation, la composition instrumentale ${ }^{1}$ et le visuel. Malgré cette richesse esthétique, le rap français voit son statut d'œuvre d'art dénié dès son apparition, où l'imaginaire médiatique assimile le rappeur avec la figure racialisée du jeune de banlieue (Hammou 2012: 85). Cet amalgame fait du rap français un produit culturel altérisé, dont la valeur réside dans sa prétendue différence sociale et raciale par rapport à la position normative (Hammou 2016: 70). La question de la valeur esthétique du rap s'imbrique donc à celle de sa réception racialisante. Au cours des années 2000 et au début des années 2010, plusieurs dépôts de plaintes, questions au gouvernement et propositions de loi visent des rappeurs en France et leur liberté d'expression. Marie Sonnette (2013: 530) montre que ces dénonciations ne retiennent que le texte des raps visés au mépris de tout élément stylistique de l'écriture, de la composition musicale ou de la mise en scène visuelle d'un clip.

2 On retrouve cette attention particulière au mode écrit dans les travaux académiques sur le rap en France, au point que l'on peut parler de «textualisation» du rap pour décrire cette réduction de ses œuvres et performances esthétiques à des paroles analysables sous leur forme écrite (Carinos et Hammou 2017 : 272). Prenant pour acquis qu'une performance rappée a un caractère politique, nombre de recherches reproduisent et naturalisent l'idée selon laquelle le rap, par le biais de ses textes, agirait d'une façon critique dans la société et aurait des effets subversifs. Les études de performance sont bien placées pour dénaturaliser ce rapport entre esthétique, textualisation et portée politique. Elles offrent notamment un regard critique sur le travail de repérage de signes qui donne des effets de réel aux phénomènes sociaux tels que le genre et la race. Judith Butler ([1990] 2006: 33) conceptualise la notion de 
performativité à travers le genre comme, « la stylisation répétée du corps, un ensemble d'actes répétés qui se fige dans le temps pour produire l'apparence de la substance, d'un état d'être naturel ». Les travaux de Nicole R. Fleetwood inscrivent la notion de performativité dans les critical race studies. Pour Fleetwood (2011:6), les discours raciaux dépendent d'un sujet intelligible, visible et agissant pour faire sens. Elle considère que le fait de repérer les signes visibles d'une prétendue différence raciale et d'en faire sens comme indicateurs d'une race, produit en effet la race. Ce «travail» de décodage est un acte performatif qui réalise la différence raciale ${ }^{2}$.

3 L'acte d'interpréter le style, l'ambiance, ou la thématique d'un objet culturel comme dénotant une identité raciale comporte aussi des effets performatifs qui naturaliseraient la race. Le lien se révèle d'autant plus clair si nous abordons l'esthétique comme un régime d'expérience sensorielle qui détermine ce qui nous est perceptible et la racialisation comme un processus qui naturalise la différence raciale en la rendant discernable à nos sens (Bishop et Roth 2019 : 3). L'analyse interne, si elle prête attention aux différentes possibilités d'appropriation de la forme esthétique, permet d'interroger le rapport entre mécanismes de racialisation et expériences sensorielles de l'objet culturel. Il ne s'agit pas de voir des objets culturels comme étant des représentations transparentes d'une réalité sociale prédéterminée qui serait la race, mais de voir comment ces objets mettent en scène la manière dont la race prend forme dans notre champ de perception (ibid.).

4 Dans cet article, il s'agit d'interroger le lien entre performance artistique et performativité à travers le morceau «Créature ratée » de Casey, qui se confronte au discours colonial et raciste par plusieurs dimensions esthétiques: celle des paroles, celle de la voix, celle de l'instrumental, celle du clip et celle d'une performance sur scène à Montreux le 9 juillet 2017. Casey est une rappeuse française d'origine martiniquaise née à Rouen et longtemps habitante de la Seine-Saint-Denis. Les presses spécialisées et généralistes saluent sa "plume acerbe» (Binet 2020) et son "sens de l'invective » (Anthokadi 2010) qu'elle applique à ses thèmes de prédilection : l'histoire coloniale de la France, le racisme de la société actuelle et la corruption de l'industrie musicale. Je cherche à démontrer comment la tension entre les aspects textuels, sonores et visuels de "Créature ratée » met en relief l'ambivalence du processus de racialisation et le régime visuel qu'il cherche à mettre en place. Pour ce faire, je propose de tenir à distance, le temps de l'analyse, certaines attentes sociales et politiques que l'on pourrait avoir du rap et de sa vocation critique supposée. Mon intention n'est pas de contester le rôle que les expériences de Casey en tant que femme noire pourraient jouer dans son processus de création artistique, ni de minorer son engagement politique, mais de ne pas faire de ces informations contextuelles et biographiques le centre de l'analyse, au détriment des techniques esthétiques qu'elle mobilise.

5 Ma démarche s'inspire du «formalisme stratégique » mis en avant par l'historien de l'art Darby English (2007: 32). English critique la tendance à voir dans l'œuvre d'un artiste noir la confirmation d'un thème, d'une démarche ou d'une prise de position prédéterminée prêtée à l'artiste en raison de son identité raciale présumée. Face à cette réception, il propose un formalisme stratégique qui s'intéresserait à tous les contextes dans lesquels les particularités d'un objet d'art et de ses techniques esthétiques prennent sens, au-delà du cadre racialisant. Pour English (34), dans les régimes dominants de réception, «la conception remplace trop souvent la perception, ce qui a 
pour effet de suspendre la vie esthétique de l'objet et de la remplacer par une vie sociale prédéterminée ${ }^{3}$ ». En ce sens, je cherche à saisir la vie esthétique de "Créature ratée " de Casey, en m'attachant à l'interaction productive entre les paroles, l'instrumental, la voix et le clip et aux possibilités d'interprétation que crée cette interaction.

6 À travers cette approche multi-sensorielle, je cherche à m'éloigner de la textualisation du rap. En prenant en compte l'interaction entre plusieurs éléments esthétiques de la performance, je m'appuie sur des travaux qui explorent les possibilités politiques du rapport artiste-interprète à travers la forme langagière du morceau, telles que les interpellations de l'auditeur et les jeux de pronoms (Pecqueux 2007, Sonnette 2015). Ma démarche se rapproche également de ce qu'Anthony Pecqueux (2009: 150) appelle « l'écoute-en-action ». "L'écoute-en-action » propose d'analyser la signification d'une chanson telle qu'elle émerge lors de l'écoute et ensuite de confronter cette signification aux usages sociaux ou politiques de la chanson. Cette approche reconnaît la possibilité de plusieurs types d'écoute et examine comment la forme du morceau rend disponible ces interprétations multiples. Pour que l'accent reste sur la possibilité de perceptions variées, j'envisage ici deux catégories d'auditeur: l'auditeur amateur et l'auditeur novice. L'auditeur amateur serait en mesure de compléter l'interprétation de la performance par sa connaissance du parcours de l'artiste et des particularités stylistiques de son œuvre, alors que l'auditeur novice découvrirait l'artiste lors de l'écoute. Cette méthode permet de mieux saisir les subtilités formelles de la performance, leurs effets, et de maintenir au centre de l'analyse non les intentions de l'artiste, mais le travail d'interprétation qui se déroule entre la performance et le public.

7 Avant de procéder à l'analyse de l'œuvre, j'aborderai dans un premier temps théorique l'intersection entre l'écoute et la racialisation comme processus performatifs audiovisuels. Il s'agira de mettre en lumière l'ambivalence et l'instabilité de ces deux processus avant de démontrer la manière dont la performance de Casey les exploite pour mettre l'auditeur devant un travail interprétatif complexe. L'analyse esthétique qui suivra abordera "Créature ratée » à quatre niveaux performatifs. D'abord, il s'agira de démontrer comment les figures de style mettent en scène la façon dont le regard racialisant opère lors de la rencontre entre le colon et la personne colonisée. Deuxièmement, l'analyse se portera sur le jeu de pronoms et la manière dont la rappeuse interpelle l'auditeur. Puisque le morceau reproduit le discours colonial à la première personne, il s'agit de voir quels rapports la structure des paroles rend disponibles sur le plan moral et affectif. Je consacrerai le troisième temps de l'analyse esthétique à la façon dont l'instrumental et l'interprétation vocale invitent l'auditeur à inverser la signification des paroles. Dans l'ultime étape de l'analyse interne, j'aborderai le clip réalisé par Chris Macari ainsi que la performance sur scène, afin de voir comment la mise en visibilité de la rappeuse confronte le spectateur au non-dit de la performance et, par conséquent, à l'urgence d'établir un pacte de solidarité avec la rappeuse et de rejeter la voix narrative des paroles. 


\section{L'écoute et la racialisation comme phénomènes audiovisuels}

Dans cette première partie théorique, j'examinerai les particularités esthétiques du rap au croisement des modes textuels, sonores et visuels. Cet engagement multi-sensoriel peut introduire certains mécanismes de racialisation lors de l'expérience de l'écoute. Comment l'interaction entre les paroles, la voix et le mode visuel façonne-t-elle notre rapport à la musique et à l'artiste? Pour Christian Béthune (2004: 81), le flow, ou l'interprétation vocale, fait que le texte devient "poésie en acte». La voix anime un texte de rap et lui donne sa dimension vivante, ses effets de présence. Anthony Pecqueux (2007: 108) détaille comment les rappeurs créent ces effets de présence à travers l'auto-ironie, ou des méta-commentaires sur le rap qu'ils sont en train de produire et l'interpellation directe de l'auditeur. Pour Pecqueux, cela met en place un rapport quasi corporel entre le rappeur et l'auditeur, en donnant l'impression que le corps rappant est placé devant celui qui l'écoute. L'écriture de la voix qu'est le rap renforce cette dimension corporelle de l'écoute, dans le sens où les sonorités de cette écriture représentent "une mimétique scénique des pulsions et des états corporels » (Rubin 2002: 269). La matérialité de la voix rappée porte en elle la possibilité de susciter des réponses affectives, un ressenti plus viscéral chez son auditeur qui signifie au-delà des paroles.

Alors que ces travaux précédents nous aident à comprendre comment la voix peut rendre le corps de l'artiste présent dans la perception de l'auditeur, ils ne s'interrogent pas sur la manière dont les qualités vocales peuvent rendre la présence de l'artiste intelligible à travers les prismes de race, de classe ou de genre. L'auditeur associe-t-il certains usages de langage ou timbres de voix à certaines identités? Comment ces impressions sonores aident-elles l'auditeur à imaginer l'artiste qui produit la voix rappée ? Pour Nina Sun Eidsheim $(2019: 2)$, on tient à ce que la voix révèle l'intériorité et l'essence même d'une personne. Or, un chanteur ne possède pas de qualités vocales définies une fois pour toutes; l'oreille de l'auditeur négocie et attribue du sens à la voix, en y apportant des grilles d'interprétation formées par sa propre culture et ses expériences personnelles (Eidsheim 2019: 9). Catherine Rudent (2021:14-15) défend une position analogue. Alors que la notion de "grain de la voix » telle que la développe Barthes est souvent mobilisée pour suggérer une singularité indicible de la voix et sa capacité de manifester la matérialité du corps, Rudent maintient que Barthes, en évoquant cette dimension corporelle du grain de la voix, fait référence uniquement à la matérialité de la langue qui produit différents sons. La puissance émotive ou l'urgence politique que l'on prête à une voix relèveraient donc plutôt d'une réception socialement déterminée que d'une réalité physique de tel ou tel timbre de voix (Rudent $2012: 14)$.

10 Jennifer Lynn Stoever (2016: 11) appelle le processus selon lequel on naturalise l'association entre certaines qualités vocales ou environnements sonores et des interprétations racialisantes, « la ligne de couleur sonore » (the sonic color line). La ligne de couleur sonore fait que l'oreille codifie et associe certains stimuli sonores à des corps racialisés. Pour Stoever, la ligne de couleur sonore implique des processus de différenciation et de hiérarchisation. L'identité sonore blanche se définit par rapport aux figures racialisées de l'Autre, dont l'altérité peut se faire entendre par des accents, l'usage des dialectes ou des mots d'argot, des énoncés extra-verbaux, ou bien par des 
bruits ambiants (11-12). Dans le contexte des États-Unis, Mendi Obadike (2005 : 176) démontre que l'aspect sonore a même la capacité d'évoquer la blackness sans la présence de personnes noires sur le plan visuel. Tel est le cas des films à distribution majoritairement blanche qui utilisent le rap pour se créer une ambiance décontractée, hors-la-loi et dynamique en mobilisant des stéréotypes de personnes noires.

11 Les expériences auditives peuvent donc jouer un rôle aussi important que celui des expériences visuelles dans le processus de racialisation. Le décalage, la tension et l'interaction entre ces deux modes sensoriels approfondissent notre compréhension de la vie sociale et esthétique de la race. L'analyse interne d'un objet culturel racialisé devrait donc saisir les interactions sensorielles du processus de racialisation, tout en prenant en compte l'instabilité et l'ambivalence qui y sont inhérentes. Dans le cas de l'œuvre "Créature ratée » de Casey, comment les différents éléments formels de la performance exploitent-ils le processus audiovisuel de racialisation pour mettre en scène la rencontre coloniale? À travers quelles techniques de voix ou quelles figures de style la rappeuse offre-t-elle à l'auditeur des clés pour déchiffrer son positionnement éthique par rapport aux paroles ainsi que des éventuels effets ironiques ${ }^{4}$ ? Comment la mise en visibilité de la rappeuse lors des performances sur scène et lors du clip nuancet-elle le rapport entre les paroles et l'interprétation vocale? L'interaction entre les éléments esthétiques textuels, sonores et visuels sert-elle à reproduire la racialisation ou, au contraire, dénaturalise-t-elle ce processus?

\section{Figures de style, figure de l'altérité}

Dès le premier couplet, l'écriture de Casey crée un espace imaginaire dans lequel deux figures circulent et prennent forme. "Blanc ingénieur, noir ingénu ${ }^{5}$ »; cette première ligne concise s'organise autour de la paronomase - figure de style de prédilection dans le rap (Barret 2008: 72) - qui rapproche les mots « ingénieur » et «ingénu » par leurs sonorités. Cette première opposition structure le couplet selon un registre racial et introduit donc dans l'expérience auditive un travail de visualisation racialisante. La blanchité s'associe à un savoir technique et spécialisé, tandis que la noirceur ${ }^{6}$ se voit renvoyée à la naïveté et à la crédulité. L'opposition mobilise un lexique de l'enfance, un schéma classique de racisme anti-noir (Ndiaye 2008:217). La césure renforce la dichotomie au niveau du sens, tandis que le rapprochement phonétique de la paronomase fait en sorte que ces deux images se reflètent et se définissent dans leur opposition comme dans un miroir. Le premier couplet se structure autour de ces duels antonymiques de syntagmes nominaux qui planent sans référent stable et trouvent leur sens dans le contraste. "Amateur de belles lettres, de poésies / Frappeur de tambour avec fracas et frénésie ", renforce la dimension esthétique de ces contrastes. La phase ${ }^{7}$ distingue une appréciation détachée et formelle d'objets d'art légitimes et une activité culturelle indissociable d'émotions excessives et de gestes corporels incontrôlés. Le texte invite l'auditeur à suivre une distribution de valeur qui bascule entre deux pôles. Par ce travail d'attribution, "l'inventeur cravaté » et la "créature ratée " prennent corps l'un par rapport à l'autre, aboutissant aux figures du colon et du colonisé. L'écriture de Casey esthétise donc le processus de différenciation hiérarchique dans lequel la racialisation puise sa force. du colon se mue en détenteur du regard. Une fois le regard tourné entièrement vers la 
figure du colonisé, la forme de l'écriture passe d'oppositions de syntagmes nominaux aux descriptions totalisantes sous forme de phrases verbales. Les images qui auparavant signifiaient par contraste commencent à se greffer à des corps particuliers, « leur sang est souillé / leurs yeux sont fuyants ». L'introduction de « leur » indique un glissement de positionnement du narrateur : la narration se fond dans le regard du colon. À travers ce regard, ces descriptions corporelles prennent la forme d'un constat évident et naturalisé : «Ses fesses sont une énorme masse / Conséquence, sa silhouette n'a aucune forme de classe ». Par le cumul de ces constats, la figure de la personne colonisée commence à se matérialiser dans la déformation que lui fait subir le regard du colon. En enchaînant de prétendues manifestations visibles des stéréotypes coloniaux sur les personnes noires, la structure du deuxième couplet trace le travail performatif de la race. Les images de l'écriture de "Créature ratée » font surgir de l'implicite un sujet visible, agissant et reconnaissable dont le discours racial dépend pour faire sens (Fleetwood 2011:6).

Les représentations racistes dont "Créature ratée " témoigne répètent la mise en spectacle des personnes racisées, notamment à l'occasion des zoos humains en France. Les échos de ces "spectacles" résonnent dans «Créature ratée »: "Chers clients, clientes, ne vous arrêtez pas à cette laideur criante / Les jungles de l'Afrique, lointaines et luxuriantes / nous offrent des spécimens de différentes variantes ». Casey cite la figure du monstre que les zoos humains $\mathrm{du}_{\mathrm{XIX}}^{\mathrm{e}}$ siècle proposaient comme marchandise, ainsi que les expériences affectives que ces zoos cherchaient à susciter : la peur et le dégoût, mais aussi la curiosité et le désir de l'exotique (Bancel et al. 2004 : 9). En outre, par l'accent mis sur les aspects corporels de la figure du colonisé (« ses fesses sont une énorme masse ", "sa peau, une carapace de cuir sans souplesse »), le deuxième couplet de «Créature ratée " met en lumière la manière dont ces exhibitions de l'Autre cherchaient à vulgariser une idéologie physionomique de la race (66), ainsi que le caractère fortement genré de ces fantasmes (Mitchell 2020). Néanmoins, Casey n'emploie aucun marqueur temporel dans "Créature ratée », ce qui fait du morceau un espace performatif où les violences du passé se fondent dans celles du présent. Par cette absence de division temporelle claire entre le passé colonial et le temps présent de l'écoute, "Créature ratée » s'inscrit dans une tradition critique postcoloniale du rap français des années 2000 dans laquelle certains artistes évoquent un lien entre leurs expériences vécues du racisme et l'histoire coloniale de la France (Djavadzadeh 2015, Hammou 2022).

\section{Jeux pronominaux et positionnements éthiques}

15 La structure narrative du morceau reste organisée autour du point de vue du colon. Casey prête son «je » à la figure du colon, «bon chrétien, j'acquiesce, volontiers, j'angoisse / Les os glacés, placés devant ce corps sans grâce ». À la première personne, elle témoigne de l'effroi que provoque la rencontre avec la personne colonisée. Non seulement Casey investit la figure du colon d'une intériorité sentimentale, mais sa voix la véhicule. La réalité du projet colonial vue par les personnes colonisées reste donc un non-dit au niveau des paroles. Quels rapports auditeur-interprète ce choix narratif rend-il possibles? Le fait que le « je » narratif du morceau s'aligne sur le point de vue du colon peut amener l'auditeur à remettre en question la possibilité d'établir un pacte de confiance avec la voix narrative. L'auditeur amateur serait notamment en mesure de 
mobiliser sa connaissance de l'œuvre de Casey et les morceaux critiques de la rappeuse envers le passé colonial de la France et envers le racisme pour la situer en opposition vis-à-vis du « je » narratif. L'auditeur novice pourrait se saisir des descriptions racistes telles que, «dépouillés d'humanité, leurs yeux sont fuyants ». Dans la mesure où elles sortent du domaine des usages acceptables de la langue et se dirigent vers une cible précise "eux", elles suggèrent une certaine mise à distance. Les représentations déshumanisantes qu'énonce la voix narrative mettraient ainsi l'auditeur - et tout particulièrement l'auditeur novice - face à ses propres convictions morales et le pousseraient à placer la rappeuse dans la même position éthique afin de pouvoir poursuivre l'écoutes.

Alors que Casey assume le «je » du colon, la place proposée à l'auditeur reste plus ambivalente. Lors du premier couplet, la rappeuse emploie l'impératif à la première personne du pluriel, « apprenons-leur à prier Jésus-Christ sans crier / Et trier la canne à sucre, tout en souriant ». Si l'auditeur se sent concerné par l'impératif de la rappeuse, il se retrouve complice de la mission civilisatrice et esclavagiste. Mais le refrain exclut directement l'auditeur de cet entre-soi colonisateur, en l'interpellant depuis une autre place, «toi, ton identité, c'est d'être la créature ratée ». Ce genre d'interpellation violente est, selon Pecqueux (2007 : 134), « la plus risquée mais aussi la plus efficace des façons d'attirer l'attention auditive, dans la mesure où l'auditeur doit ouvrir une enquête interprétative afin de s'assurer que c'est bien l'autre que lui qui est ainsi violenté ». Dans son analyse du morceau «Dans nos histoires » de Casey, Karim Hammou (2020 : 391) montre que les techniques d'interpellation de la rappeuse mettent l'auditeur face aux enjeux éthiques de son écoute et maintiennent la possibilité d'une multiplicité de réactions, au risque de mettre en danger le rapport artiste-interprète. Dans le cas de "Créature ratée ", reconnaître l'autre dans l'interpellation du refrain serait soutenir implicitement l'acceptabilité de ce discours déshumanisant, ainsi que l'autorité de la voix narrative. La structure des paroles et ce jeu de pronoms installent ainsi le doute envers le « je » narratif, troublant le lien entre l'artiste et l'auditeur.

\section{Interprétation vocale et attribution d'effets d'ironie}

17 Les formes d'interpellation présentes dans les paroles de "Créature ratée » invitent à une écoute attentive, critique, voire investigatrice. L'espace de négociation de sens qui s'ouvre entre l'artiste et l'auditeur crée la possibilité d'inverser la signification des paroles. En tant que mécanisme rhétorique, l'ironie comporte une dimension performative, dans la mesure où elle ne se résume pas aux intentions du locuteur, mais se réalise dans l'espace dynamique entre le locuteur et celui ou celle qui l'interprète (Hutcheon 1995 : 43). Dans cette partie, j'analyserai la manière dont les techniques de la voix et l'ambiance musicale s'imbriquent dans l'oreille de l'auditeur pour situer Casey en opposition à ses paroles et décoder des éléments d'ironie. L'auditeur amateur, faisant déjà partie du public de Casey et donc de sa communauté discursive, aurait à disposition des éléments contextuels et biographiques pour co-construire le dispositif ironique de la performance. Penser la possibilité d'une écoute novice permet plutôt d'examiner comment les techniques vocales de Casey - des changements de registre et de flow ainsi que des effets d'accumulation, de répétition ou d'écho - et leur interaction avec l'instrumental permettent d'attribuer la signification opposée au discours colonial reproduit dans les paroles. 
18 L'instrumental de "Créature ratée ", faite par Laloo, imbrique des notes graves de piano, des notes staccatos de violon et des notes aiguës synthétiques imitant une guitare électrique sur le fond d'une boîte à rythmes de batterie. Les notes aiguës, un des éléments les plus saillants de l'instrumental à côté de la batterie, introduisent un effet presque déstabilisant et hypnotisant. Cette boucle va et vient de manière cyclique. Les mesures où elle est absente confrontent l'auditeur plus brutalement aux paroles. On entend plus clairement les notes de piano qui rajoutent une ponctuation menaçante à la fin de chaque phase, ainsi que les notes staccatos du violon qui rajoutent une sensation d'urgence. La monotonie de ces notes crée un effet d'accumulation, de montée en tension qui s'étend lorsque le son déformé de la guitare est réintroduit lorsque l'on arrive au refrain. La maîtrise du rythme dans le flow de Casey joue sur la montée et le relâchement de tension pour créer des impressions de précipitation ou d'illogisme dans les paroles. Le rythme accéléré lors de l'énonciation de la phase du premier couplet, "sans histoire écrite, ni récit, donc sans passé ", ainsi que le rapprochement sonore entre «écrite " et "ni récit ", font que "donc sans passé » sonne comme un débouché logique et évident. L'interprétation vocale a pour effet de minimiser ce qui est en réalité l'effacement de siècles d'histoire et d'accentuer la logique simplificatrice derrière la vision du monde du colon. Si l'oreille de l'auditeur relève ces effets de minimisation et de simplification, ils permettent l'attribution d'une certaine absurdité ou d'un manque de fiabilité aux paroles.

19 L'effet vocal d'un relâchement trompeur revient à la fin du deuxième couplet. Casey s'adresse aux clients du marché aux esclaves ou du zoo humain, " éloignez quand même vos fillettes / Parfois le sauvage plonge dans la démence / En rage, peut réduire un humain en miette / Mais le passage du fouet le ramène au silence ». Les notes staccatos $\mathrm{du}$ violon renforcent un effet de danger, d'urgence ou de panique devant la violence supposée de la personne réduite en esclavage. L'enjambement que fait Casey entre « démence » et « en rage » peut aussi évoquer un sens de débordement ou de perte de contrôle. La dernière ligne sonne donc à l'oreille comme un decrescendo, une évacuation de pression, alors que sur le plan sémantique elle témoigne d'un acte de cruauté. Encore une fois, l'interprétation vocale crée un effet de méiose qui minimise, ou bien redore d'un ton rassurant, un acte violent. Lorsque Casey prononce le mot " silence », il y a une coupure brève dans l'instrumental. Cette pause ouvre la possibilité de faire entrer dans le récit tout ce qui reste non-dit, c'est-à-dire l'expérience de la personne qui subirait cette violence. L'acte de repérer le décalage entre le dit et le nondit permet à l'auditeur de décoder l'ironie acerbe du couplet.

20 Certaines manipulations vocales faites au niveau de la production musicale facilitent aussi la co-construction de l'ironie et la remise en question de la voix narrative. Lors du premier couplet, la production entrecoupe la phase « Humble sujet du Tout Puissant, se référant au Livre Saint » de deux interjections : " espiritu sanctus » et « amen ». La voix de Casey cite le langage ritualisé de la pratique du culte catholique sous effets d'échos lointains et dans un ton solennel. Le changement de registre surjoue la dévotion religieuse du colon. Le sérieux de la voix renforce ainsi le décalage entre cette supposée humilité devant Dieu et toutes les désignations flatteuses qui précèdent: " race qui réussit ", "martyr" et "grand penseur encensé ». L'auditeur qui replacerait ces apartés pieux dans le contexte du couplet peut ainsi attribuer une certaine hypocrisie à la voix narrative. 
21 À la clôture du morceau, l'instrumental diminue et laisse place à un sample de musique martiniquaise. Le décalage en termes de styles musicaux entre cette coda et l'instrumental pourrait amener l'auditeur à y voir une forme de réponse à la performance, ou de commentaire sur celle-ci. Pour l'auditeur qui ne connaîtrait pas la musique des Antilles, cette juxtaposition de styles musicaux indiquerait néanmoins sur le plan sonore la présence d'un autre récit, d'un autre point de vue. Pour l'auditeur dont la culture musicale permettrait d'attribuer ce sample au chanteur martiniquais $\mathrm{Ti}$ Émile et à l'instrument traditionnel du tibwa, cette sorte de ponction musicale remettrait en cause plusieurs éléments des paroles, notamment l'accusation d'une absence de passé ou bien la réduction de performances musicales aux «fracas » et "frénésie ». La citation musicale renforce ainsi les effets ironiques présents dans le morceau.

\section{La visualisation du non-dit}

Jusqu'ici, j'ai abordé la manière dont l'écriture de Casey engage le travail perceptif et esthétique du processus de racialisation, ainsi que la manière dont l'interaction entre son interprétation vocale et les paroles remet en question l'autorité narrative et oriente l'auditeur vers l'interprétation d'effets ironiques. Comment la présence physique de la rappeuse, lors de la prestation sur scène ou lors du clip, influence-t-elle l'interaction entre les modes écrits et sonores ? Quel est l'impact de la mise en visibilité de la rappeuse sur les manières dont les spectateurs peuvent s'identifier à la performance ? Lors d'une performance en live observée au festival de jazz de Montreux en 2017, Casey remplace le «toi » du refrain par le «moi » et désigne du doigt son propre corps lorsqu'elle l'interprète, «moi, mon identité, c'est d'être la créature ratée ». Le «moi » du refrain crée une tension interne et une négation du «je » des couplets. Ce glissement pronominal introduit de manière frontale la question de la racialisation de l'artiste dans la performance. En se désignant comme «la créature ratée » du refrain, Casey explicite un non-dit de l'œuvre; les représentations racistes des paroles la visent aussi. La reformulation du refrain plonge les paroles - auparavant évocatrices du passé colonial et esclavagiste - dans l'immédiateté de la performance sur scène. Ceci rend la responsabilité interprétative du public encore plus pressante. L'élément de témoignage personnel et l'évocation d'une continuité entre le discours colonial et l'expérience vécue du racisme à l'époque contemporaine appellent le spectateur à établir un pacte de solidarité avec la rappeuse face à la voix narrative.

Comment les techniques filmiques du réalisateur Chris Macari interagissent-elles avec l'interprétation vocale, l'instrumental et les paroles pour encadrer la manière dont le spectateur négocie la signification de la performance? La mise en scène du clip approfondit la confrontation entre le dit et le non-dit, notamment en évoquant sur le plan visuel l'expérience des personnes colonisées sur lesquelles les paroles semblent faire silence. Dès les premières secondes, le clip nous plonge dans l'espace performatif du quimbois, une pratique religieuse qui mélange des éléments de sorcellerie, de spiritualité et de rites catholiques (Curtius et Henry-Valmore 2013 : 738). La caméra assiste à un rite dans lequel le séancier secoue rapidement sa tête tandis que les participants forment un cercle en tenant des bougies à la main (0:25). Macari mélange des plans panoramiques qui nous situent dans l'intérieur du cercle de participants au rite, des plans filmés en plongée qui nous accordent le point de vue d'un observateur 
extérieur et des images filmées en contre-plongée qui inspirent le sentiment d'être pris dans les événements spirituels en cours. L'oscillation entre l'observation et la participation, mais aussi le style du clip en noir et blanc ainsi que les égratignures sur la bande du film renvoient à la tradition du film ethnographique, un genre qui a fait l'objet de bien des critiques d'exotisme, dont la plus fameuse reste celle d'Ousmane Sembene à l'égard de Jean Rouch : "Tu nous regardes comme des insectes " (Cervoni $1982: 77)$.

Or, les performances devant la caméra du séancier et du danseur de danmyé, un art martial martiniquais qui mélange sport de combat et joutes chorégraphiées, soutiennent le regard potentiellement exotisant de la caméra. Les deux acteurs fixent fermement l'objectif qui les filme en gros plan (0:31, 0:57). Paula Amad (2013) appelle ce regard retourné une "riposte visuelle » et elle interroge l'emploi de cette technique dans les films ethnographiques. Alors qu'Amad reconnaît la possibilité que ce regard soit fétichisé comme une preuve d'humanité ou d'agency de l'Autre dans un dispositif contrôlé par le réalisateur au profit de l'image du soi du dominant, elle cite aussi cette riposte visuelle comme un contact virtuel entre le sujet filmé et le spectateur (2013 : 64). Tout comme le pacte de solidarité établi lors de la performance sur scène, ce contrat rend plus coûteux un visionnage nonchalant et met le spectateur face à son rôle éthique d'arbitrage, quant au statut des représentations mises à sa disposition.

La mise en scène du quimbois lors du clip met le spectateur face au point de vue des personnes colonisées et réduites en esclavage. La prestation de Casey devant la caméra la rapproche de la figure du séancier - une indication visuelle qu'elle assume le rôle dévolu à ce dernier : interpréter le passé, le présent et l'avenir (Curtius et HenryValmore 2013 : 738). Casey apparaît au spectateur pour la première fois devant un mur marqué avec des croix catholiques (0:39). Elle a le visage couvert de la même poudre blanche de kaolin que le séancier du rite. Dans l'espace performatif du quimbois, la poudre signifie une mise en contact avec les défunts et donc laisse entrer les témoignages omis du discours colonial du texte. Le montage du clip témoigne de la transmission de la mémoire des morts à travers la juxtaposition rapide (0:41) d'un gros plan du dos d'un danseur et d'une célèbre photographie, apparue en 1863 dans le magazine américain Harper's Weekly, de Gordon, un homme réduit en esclavage, dont le dos avait été mutilé par des coups de fouets. Le spectateur amateur reconnaîtrait cette image d'un clip précédent de Casey, «Dans nos histoires ", sorti en 2006, et l'inscrirait ainsi dans une lignée de performances audiovisuelles antiracistes de la rappeuse.

La référence au quimbois dans "Créature ratée » joue donc un double rôle performatif. D'une part, elle fait ressortir des effets spirituels dans l'espace de la performance musicale, ici la mise en contact avec les morts qui concrétise sur le plan visuel la violence du texte. D'autre part, la mise en scène du quimbois renforce le lien entre la rappeuse et les personnes colonisées qu'évoque son texte. En ce sens, la mise en scène renvoie à une démarche de construction d'identité postcoloniale qu'Alice AterianusOwanga (2017 : 196) observe dans la mobilisation des répertoires religieux dans le rap gabonais. Le spectateur peut voir dans la référence au quimbois un lien au passé, aux racines antillaises et un prolongement des tactiques de résistance à l'oppression coloniale associées à ces pratiques religieuses (Curtius et Henry-Valmore 2013 : 738). La mise en scène du quimbois fait ressortir cet aspect de lutte anticoloniale du temps passé et l'ancre dans le présent et l'avenir. 


\section{Conclusion}

Les multiples niveaux performatifs de «Créature ratée » de Casey mettent en scène la racialisation comme un processus sensoriel qui cherche à déterminer ce qui parait perceptible et significatif. En ce sens, "Créature ratée " illustre l'importance de regarder la performance rappée dans l'ensemble de ses dimensions et non seulement dans sa dimension écrite. Analyser toutes les dimensions esthétiques de la performance permet de voir comment leur synergie exploite les ambivalences de la racialisation. À travers cette approche multi-sensorielle, la perception de l'œuvre reprend une place centrale face à la conception. Au lieu de prédéterminer la portée politique d'une performance rappée en fonction des éléments biographiques de l'artiste ou des intentions supposées de ce dernier, cet article démontre que la politisation de la performance vient des techniques esthétiques et de leur interaction.

Alors que les paroles de "Créature ratée » reproduisent le discours colonial à la première personne, c'est lorsque l'on prend en compte l'instrumental, l'interprétation vocale et la mise en images (via le clip ou les performances scéniques, telle celle à Montreux en 2017), que l'on voit comment la performance met l'auditeur devant les failles du régime de représentation que le discours colonial cherche à imposer. Au niveau des paroles, l'emploi de la paronomase met en relief le travail audiovisuel de repérage de signes qui accorde à la race des effets de réel. Cette figure de style souligne notamment la dépendance du discours racial envers un sujet racialisé intelligible comme tel pour faire sens. Le jeu d'interpellations dans le morceau confronte l'auditeur aux conséquences morales de ce travail de racialisation et l'invite ainsi à ouvrir une enquête interprétative pour déterminer le positionnement éthique de la rappeuse. Au niveau de l'interprétation vocale, des effets de montée en tension, de relâchement et d'écho remettent en question l'autorité de la voix narrative. Le clip affaiblit encore plus l'autorité du discours colonial, notamment en mettant en images le non-dit de son récit, c'est-à-dire l'expérience des personnes colonisées. L'interaction entre ces différents éléments amène l'auditeur à mettre en relation le dit et le non-dit, et par conséquent à décoder des effets d'ironie et à inverser la signification des paroles. ${ }^{9}$

\section{BIBLIOGRAPHIE}

Amad, Paula, 2013, « Visual Riposte: Looking Back at the Return of the Gaze as Postcolonial Theory's Gift to Film Studies », Cinema Journal, vol. 52, n 3, p. 49-74.

Anthokadi, 2010, "Chronique, Casey : Libérez la bête », Abcdrduson, 28 mars 2010, [En ligne], https://www.abcdrduson.com/chroniques/liberez-la-bete/, consulté le 12 juillet 2021.

Aterianus-Owanga, Alice, 2017, «Le rap, ça vient d'ici ». Musiques, pouvoir et identités dans le Gabon contemporain, Paris, Éditions de la maison des sciences de l'Homme. 
Bancel, Nicolas, Blanchard, Pascal, Boëtsch, Gilles, Deroo, Éric et Lemaire, Sandrine, 2004, «Introduction : Zoos Humains : Entre mythe et réalité », dans N. Bancel et al. (dir.), Zoos humains, Paris, La Découverte, p. 5-18.

Barret, Julien, 2008, Le Rap ou l'artisanat de la rime, Paris, L'Harmattan.

Béthune, Christian, 2004, Pour une esthétique du rap, Paris, Klincksieck.

Binet, Stéphanie, 2020, «La rappeuse Casey mord encore », Le Monde, 6 mars 2020, [En ligne], https://www.lemonde.fr/culture/article/2020/03/06/la-rappeuse-casey-mordencore_6032095_3246.html, consulté le 12 juillet 2021.

Bishop, Cécile et Roth, Zoë, 2019, « Introduction: Race and the Aesthetic in French and Francophone Cultures ", L’Esprit Créateur, vol. 59, n² 2, p. 1-11.

Blanchard, Pascal, Bancel, Nicolas et Lemarie, Sandrine, 2004, « Les zoos humains : le passage d'un "racisme scientifique" vers un "racisme populaire et colonial" en Occident ", dans N. Bancel et al. (dir.), Zoos humains, Paris, La Découverte, p. 63-71.

Butler, Judith, [1990] 2006, Trouble dans le genre. Le féminisme et la subversion de l'identité, trad. Cynthia Kraus, Paris, La Découverte.

Carinos, Emmanuelle et Hammou, Karim, 2017, « Approches du rap en français comme forme poétique », dans C. Legoy et al. (dir.), La poésie délivrée, Nanterre, Presses universitaires de Paris Nanterre, p. 269-284.

Cervoni, Albert, 1982, « Une confrontation historique en 1965 entre Jean Rouch et Sembène Ousmane : “Tu nous regardes comme des insectes" ", CinémAction, n 17, p. 77-78.

Curtius, Anny Dominique et Henry-Valmore, Simonne, 2013, " Quimbois », dans P. Taylor et al. (dir.), The Encyclopedia of Caribbean Religions, vol. 2, Champaign, The University of Illinois Press, p. 738-741.

Djavadzadeh, Keivan, 2015, « Colonialité du pouvoir, postcolonialité du rap : l'émergence et la répression d'un rap français structuré autour de la critique postcoloniale dans les années 2000 », The Postcolonialist, vol. 3, $\mathrm{n}^{\circ}$ 1, p. 1-9.

Eidsheim, Nina Sun, 2019, The Race of Sound: Listening, Timbre, and Vocality in African American Music, Durham, Duke University Press.

English, Darby, 2007, How to See a Work of Art in Total Darkness, Cambridge (MA), MIT Press.

Fleetwood, Nicole R., 2011, Troubling Vision: Performance, Visuality, and Blackness, Chicago, University of Chicago Press.

Hammou, Karim, 2012, Une histoire du rap en France, Paris, La Découverte.

Hammou, Karim, 2016, « Mainstreaming French rap music. Commodification and artistic legitimation of "othered" cultural goods ", Poetics, vol. 59, p. 67-81.

Hammou, Karim, 2020, « Das Gedächtnis der Vielheit. Minderheitsuniversalismus im französischen Rap », Die Tonkunst, $n^{\circ}$ 4, trad. Gesa zur Nieden, p. 380-392.

Hammou, Karim, 2022 (à paraître), « Les traumatismes (post)coloniaux dans le rap en français : mises en musique d'une citoyenneté critique », dans S. Segler-Meßner et I. von Treskow (dir.), Traumatisme et mémoire culturelle, Berlin, De Gruyter.

Hutcheon, Linda, 1995, Irony's Edge: The Theory and Politics of Irony, Londres, Routledge. 
Mitchell, Robin, 2020, Vénus noire. Black Women and Colonial Fantasies in Nineteenth-Century France, Athens, University of Georgia Press.

Ndiaye, Pap, 2008, La condition noire : essai sur une minorité française, Paris, Calmann-Lévy.

Obadike, Mendi, 2005, Low Fidelity: Stereotyped Blackness in the Field of Sound, Thèse en littérature, Durham, Duke University.

Pecqueux, Anthony, 2007, La voix du rap. Essai de sociologie de l'action musicale, Paris, L'Harmattan.

Pecqueux, Anthony, 2009, «L'écoute-en-action : l'écoute de la chanson comme activité sociale », dans A. Pecqueux et O. Roueff (dir.), Écologie sociale de l'oreille. Enquêtes sur l'expérience musicale, Paris, Éditions de l'EHESS, p. 149-186.

Rubin, Christophe, 2002, «Le texte de rap : une écriture de la voix », Actes du $22^{e}$ colloque d'Albi, 2001, Toulouse, CALS-CPST.

Rudent, Catherine, 2020, "Oublier "The grain of the voice" : étudier la voix dans les chansons ", Volume ! La revue des musiques populaires, vol. 17, n 1, p. 7-26, [En ligne], https://doi.org/10.4000/ volume.7843.

Rudder Véronique de, Poiret, Christian et Vourc'h, François, 2000, L'Inégalité raciste. L'universalité républicaine à l'épreuve, Paris, PUF.

Sonnette, Marie, 2013, Des manières critiques de faire du rap : pratiques artistiques, pratiques politiques, Thèse de doctorat en sociologie, Paris, Université Sorbonne Nouvelle.

Sonnette, Marie, 2015, « Des mises en scène du "nous" contre le "eux" dans le rap français ", Sociologie de l'Art, $\mathrm{n}^{\circ}$ 23-24, p. 153-177.

Stoever, Jennifer Lynn, 2016, The Sonic Color Line: Race and the Cultural Politics of Listening, New York, New York University Press.

\section{Corpus}

Casey, 2006, « Dans nos histoires », mis en ligne par Casey Anfalsh, 17 janvier 2020, https:// youtu.be/_BnaXMnOWlI, consulté le 12 juillet 2021.

Casey, 2010, « Créature ratée », Libérez la bête, Anfalsh.

Casey, 2010, « Créature ratée », mis en ligne par Casey Anfalsh, 17 janvier 2020, https://youtu.be/ Fa7nN1YXQv0, consulté le 12 juillet 2021.

\section{ANNEXES}

\section{Paroles de « Créature ratée »}

Blanc ingénieur, noir ingénu

Au rang des seigneurs ou dépourvu de génie

Grand vainqueur à l'âme de gagneur ou fainéant et menteur

Tout ça dans un même corps réunit

Race qui réussit, espèce peu avancée

Sans histoire écrite, ni récit donc sans passé 
Inventeur cravaté, créateur gâté, créature ratée

Sans grandeur à cravacher

Civilisateur sensibilisé par train d'vie aisé, hectares à dévaliser

Martyr dans la moiteur des alizés, moteur d'immenses fortunes réalisées

Amateur de belles lettres, de poésies

Frappeur de tambour avec fracas et frénésie

Grand penseur encensé, petite frappe assassin, baroudeur résistant, quémandeur assisté

Humble sujet du Tout Puissant (espiritu sanctus) se référant au Livre Saint (amen)

Individus indécent qui dissimulent à peine et leur sexe et leurs seins

Et leur sang est souillé, leurs chants sont bruyants

Dépouillés d'humanité, leurs yeux sont fuyants

Apprenons-leur à prier Jésus-Christ sans crier

Et trier la canne à sucre, tout en souriant

Oui, la nature a fauté

S'est plantée en beauté

Toi, ton identité

C'est d'être la créature ratée

La nature a fauté

S'est plantée en beauté

Toi, ton identité

C'est d'être la créature ratée

Que disent la science et la messe

Toutes les grandes puissances du monde et la presse

Que cette noirceur en présence dans la pièce

Nous est inférieur, comme la chienne et l'anesse

Bon chrétien, j'acquiesce, volontiers, j'angoisse

Les os glacés, placés devant ce corps sans grâce

Et ses cuisses sont trop grosses

Ses narines embrassent, chacune des extrémités de sa pauvre face

Ses fesses sont une énorme masse

Conséquence, sa silhouette n'a aucune forme de classe

Sa peau, une carapace de cuir sans souplesse

Lèvres si épaisse qu'elles en deviennent grimace

La pommette est saillante

Chers clients, clientes ne vous arrêtez pas à cette laideur criante

Les jungles de l'Afrique, lointaines et luxuriantes

Nous offrent des spécimens de différentes variantes

Leurs femelles sont fertiles et vaillantes

Docile et idiote, font-elles mêmes leurs paillotes

Les mâles ont côtoyé éléphants et coyotes

Nourries de chasse, de pêche et de cueillette

Éloignez quand même vos fillettes

Parfois le sauvage plonge dans la démence

En rage, peut réduire un humain en miette

Mais le passage du fouet le ramène au silence... 
Oui, la nature a fauté

S'est plantée en beauté

Toi, ton identité

C'est d'être la créature ratée

La nature a fauté

S'est plantée en beauté

Toi, ton identité

C'est d'être la créature ratée

La créature ratée $(\times 4)$

Être, la créature ratée

\section{NOTES}

1. Je qualifierai « composition musicale » d'« instrumental » dans la suite de cet article, selon le terme en vigueur dans le monde du rap.

2. Dans cet article, j'aborderai la race selon cette conception: non pas en tant que réalité biologique, mais en tant que phénomène social qui prend des effets de réel. Le terme racialisation fait référence au processus social qui attribue une identité raciale à une personne ou à un groupe de personnes. Le terme racisme renvoie à la catégorisation et à la hiérarchisation de groupes humains. Voir de Rudder, Poiret et Vourc'h (2000).

3. «Conception too often replaces perception, in effect suspending the aesthetic life of the object and replacing it with a predetermined social one. »

4. Je reviendrai plus longuement sur la dimension ironique au fil des analyses, car au lieu d'être un dispositif prédéterminé par les intentions de l'artiste, l'ironie resurgit surtout lors de la négociation de sens entre l'œuvre et l'auditeur.

5. La retranscription intégrale des paroles se trouve en annexe.

6. Au lieu d'employer le mot anglais blackness, je choisis sa traduction française, " noirceur ", employée par Casey lors du deuxième couplet et courante dans les travaux de critical race studies en français (voir notamment Frantz Fanon, Peau noire, masques blancs, Paris, Seuil, [1952] 1995).

7. Dans le vocabulaire du rap, la phase est l'équivalent du vers poétique.

8. «Créature ratée » représente donc un contre-exemple à la règle prescriptive avancée par Pecqueux (2007: 72) selon laquelle le rappeur serait toujours à la fois auteur, interprète et protagoniste.

9. Je tiens à remercier Karim Hammou pour ses précieux conseils et relectures. 


\section{RÉSUMÉS}

Cet article examine comment les particularités esthétiques du rap peuvent mettre en scène des rapports sociaux de pouvoir. Il s'agit d'analyser le morceau «Créature ratée » de la rappeuse Casey à l'échelle des paroles, de l'interprétation vocale, de la production musicale, de la performance sur scène et du clip afin d'analyser comment ces formes travaillent ensemble pour dénaturaliser le regard racialisant. Les paroles de "Créature ratée " reproduisent le discours colonial à la première personne et mettent donc l'auditeur devant un travail interprétatif important et créent un rapport auditeur-interprète complexe. L'interaction productive entre les différents éléments de la performance invite à mettre en relation le dit et le non-dit des paroles, et par conséquent à inverser leur signification. À travers l'imbrication des paroles, de la voix et du visuel, «Créature ratée » vide le discours racial de l'intérieur, en montrant ses ambivalences, la fausseté de sa prétendue objectivité et sa dépendance vis-à-vis de l'Autre pour faire sens.

This article examines how the aesthetic particularities of rap music can stage social power dynamics. It analyzes the song "Créature ratée" by the rapper Casey at the level of its lyrics, vocal performance, instrumental, live performance, and music video in order to study how these forms work together to denaturalize the racializing gaze. The lyrics of "Créature ratée" reproduce colonial discourse in the first person, and thus create important interpretative work for the listener, as well as a complex listener-artist relationship. The productive interaction between the different elements of the performance invites the listener to bring together the said and the unsaid of the lyrics, and consequently inverse their signification. Through the imbrication of the lyrics, the voice, and the visual mode, "Créature ratée" empties out racial discourse from the inside, showing its ambivalences, the falseness of its supposed objectivity, and its total dependence on the Other in order to find meaning.

\section{INDEX}

Mots-clés : rap, Casey, esthétique, race, performativité, analyse interne

Keywords : rap, Casey, aesthetics, race, performativity, close reading

\section{AUTEUR}

\section{EMILY Q. SHUMAN}

New York University 